OPEN

SUBJECT AREAS:

OLFACTORY RECEPTORS

MOLECULAR BIOLOGY

CELL BIOLOGY

OLFACTORY BULB

Received

15 May 2013

Accepted

7 June 2013

Published

24 June 2013

Correspondence and requests for materials should be addressed to S.I.K. (sigrun. korsching@uni-koeln.

de)

* Current address:

Research Center for

Child Mental

Development,

University of Fukui, 23-

3 Matsuoka-

shimoaizuki, Eiheiii,

Fukui 910-1 193,

JAPAN.

\section{Zebrafish crypt neurons project to a single, identified mediodorsal glomerulus}

\author{
Gaurav Ahuja', Ivan Ivandić', Mehmet Saltürk', Yuichiro Oka'*, Walter Nadler² \& Sigrun I. Korsching'
}

'Institute of Genetics, University at Cologne, D-50674 Cologne, Germany, ${ }^{2}$ Institute for Advanced Simulation (IAS), Juelich Supercomputing Centre (JSC), Forschungszentrum Juelich, D-52425 Juelich, Germany.

Crypt neurons are a third type of olfactory receptor neurons with a highly unusual "one cell type - one receptor" mode of expression, the same receptor being expressed by the entire population of crypt neurons. Attempts to identify the target region(s) of crypt neurons have been inconclusive so far. We report that TrkA-like immunoreactivity specifically labeled somata, axons, and terminals of zebrafish crypt neurons and reveal a single glomerulus, mdg2 of the dorsomedial group, as target glomerulus of crypt neurons. Injection of a fluorescent tracing dye into the mdg2 glomerulus retrogradely labeled mostly crypt neurons, as assessed by quantitative morphometry, whereas no crypt neurons were found after injections in neighboring glomeruli. Our data provide strong evidence that crypt neurons converge onto a single glomerulus, and thus form a labeled line consisting of a single sensory cell type, a single olfactory receptor and a single target glomerulus.

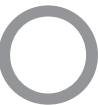

lfactory coding in vertebrates employs a receptotopic map in the target region of olfactory receptor neurons, the olfactory bulb ${ }^{1,2}$. The monogenic expression of large olfactory receptor gene families in ciliated olfactory receptor neurons engenders a correspondingly large repertoire of target modules (glomeruli) in the olfactory bulb, due to axonal convergence. Similarly, microvillous neurons, another type of olfactory receptor neurons, express large gene families and converge into many target glomeruli. Crypt neurons constitute a third type of olfactory receptor neurons ${ }^{3}$. They appeared early in vertebrate evolution, are already present in cartilaginous $\mathrm{fish}^{4}$, and have been described in many teleost fish as well ${ }^{5}$. Crypt neurons have been originally identified by their conspicuous morphology, which includes a large globular soma, the presence of both cilia and microvilli, and the eponymous crypt of unknown significance. A single olfactory receptor, the V1Rrelated ORA4, was found to be expressed in zebrafish crypt neurons ${ }^{6}$, but it is unclear whether crypt neurons project to a single target glomerulus in the olfactory bulb in accordance with the rules found for olfactory receptors of the $\mathrm{OR}^{7}$ and $\mathrm{TAAR}^{8}$ families, or whether they might connect to several target regions like neurons expressing mammalian V1Rs?.

Crypt neurons constitute an intriguing cell population, and several attempts have been made to elucidate their function ${ }^{10-13}$ and their target region in the olfactory bulb ${ }^{14-18}$. However, results have been partially incongruous, and progress has been hampered by the paucity of available markers, compounded by the absence of quantitative measures to identify crypt neurons. Germana et al $(2004)^{19}$ observed S100-like immunoreactivity in morphologically identified crypt cells, and this antibody was used in several subsequent studies, e. ${ }^{14-16}$. These attempts to use S100-like immunoreactivity as marker for crypt neurons have led to the suggestion that crypt neuron terminals are located in the dorsomedial and lateral glomerular fields of the olfactory bulb ${ }^{14}$. However, anti S100 antibody requires very particular assay conditions to serve as specific marker for crypt neurons ${ }^{6}$, which were not met in those studies, resulting in additional labeling of numerous receptor neurons with microvillous morphology and corresponding uncertainty about the true target glomeruli of crypt neurons. Oka et al, $2012^{6}$ could show that the microvillous-like subpopulation labeled by the S100 antibody indeed expressed an $s 100$ gene, $s 100 z^{20}$, whereas the immunoreactivity in crypt neurons was caused by an unknown protein with better retention in the tissue, allowing to differentiate between these two cell populations by omitting the fixation step of the standard immunohistochemical procedure. However, this modification precludes the use of S100-like immunoreactivity in many procedures, including visualization of axonal terminal regions.

Another marker reported for crypt neurons, anti-trkA antibody ${ }^{21}$, has not been investigated further so far. Using population-based quantitative analysis we report here that trkA-like immunoreactivity constitutes a robust 
and sensitive marker for crypt neurons that reliably labels only crypt neurons in a variety of experimental conditions. Using this marker we could identify a single mediodorsal glomerulus, mdg2, as target glomerulus for crypt neurons. Backtracing from this glomerulus with the fluorescent tracer DiI labeled mostly crypt neurons, in contrast to backtracing from neighboring glomeruli, confirming mdg2 as target glomerulus for crypt neurons. These results are consistent with a 'one olfactory receptor cell type - one target glomerulus' concept, a novel coding strategy in vertebrate olfaction.

\section{Results}

TrkA-like immunoreactivity is a robust and sensitive marker for crypt neurons. Crypt neurons are an intriguing olfactory receptor neuron population of so far unclear function. Here we have performed immunohistochemistry with TrkA antibody on cryostat sections of adult zebrafish olfactory epithelium to examine the suitability of TrkA-like immunoreactivity as a marker of crypt neurons under standard histological conditions, i.e. in fixed tissue. We identify crypt neurons in fixed tissue by a quantitative analysis of cell shape and position, using the corresponding values measured for an established crypt neuron marker ${ }^{6}$ as reference.

In fresh frozen tissue sparse globose cells are co-labeled by $\mathrm{S} 100$ and TrkA antibody (Fig. 1d). In quantitative evaluation we find that TrkA-label completely overlaps with S100-label in unfixed tissue (Fig. 1e). In other words, TrkA replicates the S100 staining under conditions, in which S100-labeling is specific to crypt neurons, $c f .^{6}$.

Moreover, TrkA labeling also is restricted to sparse large globose cells under standard histological conditions (Fig. 1a-c, f, g), in which the S100 antibody labels many additional cells (Fig. 1c, e-g). As a measure of globosity we chose the ratio of vertical to horizontal diameter (SI Fig. 1a), $c f .6$. The distribution of values for this ratio is undistinguishable for cells labeled by $\mathrm{S} 100$ antibody in unfixed tissue and TrkA antibody-labeled cells in either fixed or unfixed tissue (Fig. 1f-g). Thus, TrkA-labeled cells in fixed tissue exhibit very similar cellular morphology to bona fide crypt neurons ${ }^{6}$ (Fig. 1f, g). In contrast, values for the diameter ratio of S100-labeled cells in fixed tissue deviate drastically (Fig. 1f-g). Pairwise comparisons of the unbinned distributions by a Kolmogorov-Smirnov test ${ }^{22}$ showed $\mathrm{p}$ values above 0.5 for the conditions 'TrkA in fixed tissue', 'TrkA in unfixed tissue', 'S100 in unfixed tissue', whereas all comparisons with 'S100 in fixed tissue' exhibited p values below $10^{-6}$ (SI Table 1). Thus, consistent with previous reports ${ }^{6}$, statistical evaluation shows the population of cells labeled with S100 antibody in fixed tissue to be significantly different from the crypt neuron population, due to the presence of a large additional cell population with more elongated shapes. In contrast, TrkA-like immunoreactivity is a specific marker for crypt neurons, both in unfixed and in fixed tissue.

Furthermore, TrkA-labeled cells in fixed tissue exhibit an apicalcentered distribution (Fig. 1a-b,h-i) characteristic for bona fide crypt neurons, $c f .^{6}$. Again, the distribution for S100-labeled cells in fixed tissue (Fig. 1h-i) is significantly different $(\mathrm{p}<0.0002)$, even though the S100-labeled non-crypt cells are rather apically located as well. Note that the Kolmogorov-Smirnov test detects small differences in position between different cell populations ( $c f$. Fig. 1i), which are easily overlooked when relying on qualitative inspection or even the common histogram representation (Fig. 1h). We observe roughly 400 TrkA-labeled cells per olfactory epithelium, in good accordance with previously published values for frequency of crypt neurons ${ }^{6}$. Taken together, we conclude that TrkA-like immunoreactivity is a reliable, robust and specific marker for crypt neurons.

TrkA-like immunoreactivity is not caused by TrkA protein. To establish, whether TrkA-like immunoreactivity measures TrkA protein, we have compared the mRNA expression pattern of TrkA with the immunolabeling. An intron-spanning primer pair for TrkA was made within the region common to all predicted isoforms ( $c f$. Ensembl gene ENSDARG00000004586) and expression was examined by RT-PCR with cDNA from different tissues. Brain, known to express both $\operatorname{trk} A$ and its ligand, $\mathrm{NGF}^{23,24}$, gave a clear signal at the expected molecular weight, and nonneuronal tissues were negative as expected, but no band was detectable in olfactory epithelium (Fig. 2a).

We then performed in situ hybridization with a trkA probe on larval zebrafish whole mounts. As expected ${ }^{25,26}$, the inner ear showed trkA expression, and the same structures were labeled by the trkA antibody (Fig. 2c,d,g,h), confirming the suitability of both probe and antibody. However, no in situ hybridization signal was observed in the olfactory epithelium (Fig. 2e, i), although the Trk antibody stained one to a few globose cells per larval nose (Fig. 2f, j), in accordance with expectations for the abundance of crypt neurons at that developmental stage ${ }^{27}$.

Finally, we performed a Western blot with protein extracts from olfactory epithelium and brain (Fig. 2b) and observed a band with the characteristic fuzzy appearance of glycoproteins in brain samples at $133 \mathrm{kDa}$ (Fig. 2b), which is very similar to the apparent molecular weight reported for glycosylated TrkA ${ }^{28}$. Such a band was absent from olfactory epithelium, which, however, showed several other bands not corresponding to TrkA (Fig. 2b). Taken together, these results suggest that in olfactory epithelium TrkA-like immunoreactivity is caused by a cross-reacting protein instead of TrkA itself. The molecular nature of this antigen is unknown.

A single mediodorsal glomerulus, mdg2, is labeled by TrkA antibody. The subcellular distribution of the TrkA antigen is rather homogenous, and even the initial axon segment of individual crypt neurons is visible at high magnification (Fig. 1b). Within the olfactory bulb axons are expected to converge into common fascicles and terminal structures, which should facilitate the detection of crypt neuron target glomeruli. Hence we used the TrkA antibody for immunohistochemical labeling of whole mounts of olfactory bulb in an attempt to identify the target region(s) of crypt neurons. We report that within each olfactory bulb a single terminal structure with the typical morphology of a glomerulus is labeled by the TrkA antibody (Fig. 3a-c, SI Fig. 1c). This glomerulus is bilaterally symmetrical for the left and right olfactory bulb, but there is no mirror glomerulus within each olfactory bulb, in contrast to such patterns in the rodent olfactory bulb ${ }^{29}$. This finding is consistent with the absence of a recognizable symmetry axis in the glomerular pattern within each olfactory bulb in zebrafish ${ }^{30}$.

The TrkA-labeled glomerulus is situated extremely dorsal, as seen in cross sections (Fig. 3e), about one glomerular diameter away from the midline (Fig. 3a,d-f) and very far posterior, as seen in a dorsal view (Fig. 3a,d,f). This position was reproducibly found in ten glomeruli from five different animals, and the coordinates for the center of the glomerulus in dorsal view were quantified as $0.069+/-0.006$, $0.27+/-0.02$ (mean $+/-\mathrm{SEM}, \mathrm{n}=10$; values represent normalized distance from the dorsal posterior end of the olfactory bulb and from the midline, respectively, see SI Fig. 1f,g for a graphical definition of coordinates). The shape of the glomerulus is oblong (majorto-minor diameter ratio $1.50+/-0.07$, mean $+/-\mathrm{SEM}, \mathrm{n}=10$ ), with the long axis parallel to the telencephalic surface and its dimensions $(50$ to $100 \mu \mathrm{m})$ are within the range reported for other glomeruli in zebrafish ${ }^{30}$. In the majority of cases two axon fascicles enter the TrkA-labeled glomerulus (Fig. 3a-c). Convergence of TrkAlabeled axons seems to occur well before they reach the target region proper, because the nerve bundles are visible for long distances (Fig. 3a,d,f) similar to observations made for genetically labeled glomeruli ${ }^{7,9,31}$

Zebrafish olfactory glomeruli form a stereotyped pattern and are interindividually recognizable ${ }^{17,30}$. We have performed double labeling of TrkA and SV2, a synaptic marker labeling the complete glomerular pattern, to identify the TrkA glomerulus (Fig. 3 a,d,f). We 

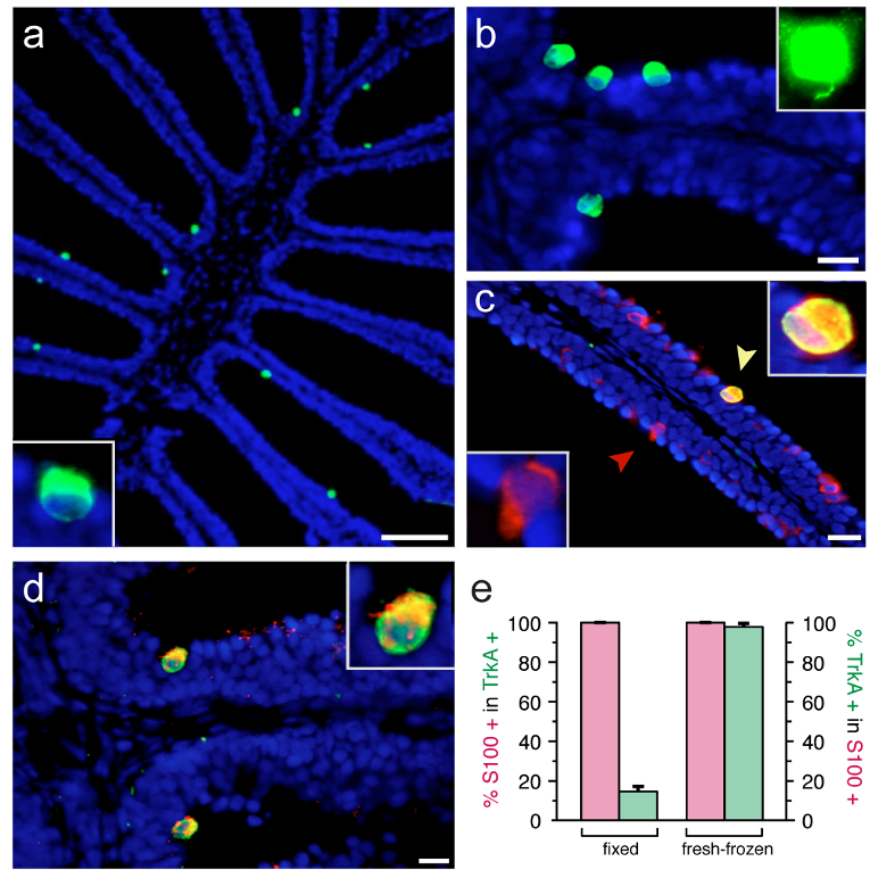

e
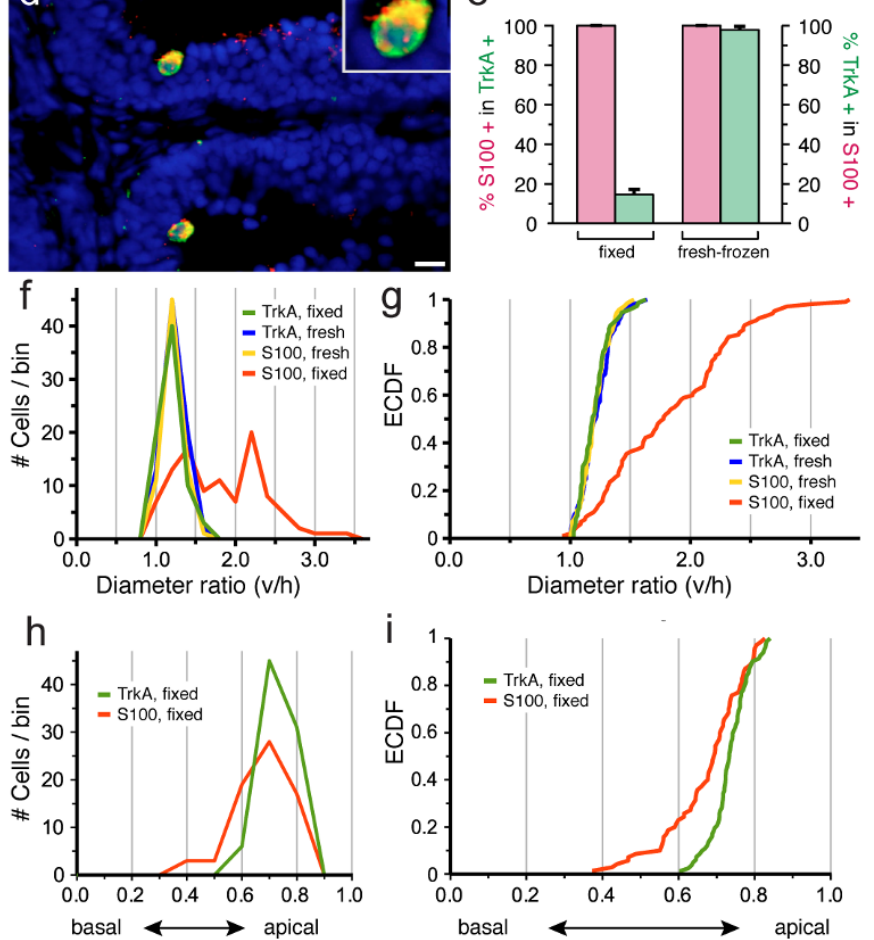

Figure 1 | TrkA-like immunoreactivity co-localizes with S100-like immunoreactivity and crypt neuron morphology. (a) TrkA-like immunoreactivity (green) is seen in a sparse population of ovoid cells in horizontal sections of olfactory epithelium (fixed); the inset shows another TrkA-labeled neuron at higher magnification. (b) Enlargement of several TrkA-labeled cells from another section showing the typical ovoid shape of crypt neurons; the inset shows another TrkA-labeled neuron at $1000 \times$ magnification, enabling visualization of the initial axon segment. (c) Double labeling with anti-S100 antibody (red) and anti-TrkA antibody (green) in fixed tissue shows TrkA labeling restricted to a subset of cells with S100-like immunoreactivity. Note the difference in morphology between double-labelled cell (yellow) and TrkA-/S100+ cells (red). (d) In fresh-frozen tissue S100-like immunoreactivity and TrkA-like immunoreactivity label the same, sparse population of globose cells. (e) Quantification of overlap between S100 and TrkA-staining, values given are mean $+/-$ SEM. TrkA-positive cells show nearly complete overlap with $\mathrm{S100-positive} \mathrm{cells} \mathrm{in} \mathrm{fresh-frozen} \mathrm{tissue}(\mathrm{p}=0.2$, Student's ttest, two-sided, unpaired), where S100 label is specific for crypt neurons ${ }^{6}$, but constitute only a minority of S100-positive cells in fixed tissue ( $\mathrm{p}<10^{-12}$, Student's t-test, two-sided, unpaired), in which $\mathrm{S} 100$ antibody labels many more cells besides crypt neurons ${ }^{6}$. (f, g) The ratio of vertical to horizontal diameter, 'diameter ratio $(\mathrm{v} / \mathrm{h})$ ', was measured for TrkA- and S100-labeled cells under standard histological conditions ('fixed') and in fresh frozen tissue ('fresh'). (f) histogram, (g) empirical cumulative distribution function (ECDF) of the unbinned distributions. Note the extreme similarity of the distribution for TrkA in both conditions with the distribution for S100 in fresh-frozen tissue and the drastic difference ( $\mathrm{p}<10^{-6}$, Kolmogorov-Smirnov test, $c f$. SI Table 1) to the distribution of S100-labeled cells under standard histological conditions ('fixed').

(h), (i) The relative height of labeled cells within the olfactory lamella was compared for TrkA- and S100-labeled cells under standard histological conditions, i.e. in fixed tissue. (h) histogram, (i) empirical cumulative distribution function (ECDF) of the unbinned distributions. The difference between distributions was highly significant (KolmogorovSmirnov test, $\mathrm{p}<0.0002)$. Scale bars $80 \mu \mathrm{m}$ for $(\mathrm{a})$ and $10 \mu \mathrm{m}$ for $(\mathrm{b}-\mathrm{d})$, respectively.

report that the TrkA-labeled glomerulus unambiguously maps to mdg2 (nomenclature after ${ }^{17}$ ), one of six glomeruli in the mediodorsal cluster. This assignment is based on nearly identical coordinates of the trkA-labeled glomerulus (cf. Fig. 3e, f, SI Fig. 1f,i) and mdg2 (SI Fig. 1f,g,j, $c f .{ }^{17}$ ) in all three dimensions (anterior-posterior, mediallateral, and dorsal-ventral). All neighboring glomeruli show clearly different coordinates (SI Fig. 1g,i,j). Previously this mediodorsal cluster as well as a dorsolateral region had been suggested as target regions for crypt neurons, albeit based on S100 antibody staining in unspecific mode, e.g. ${ }^{14}$. We did not detect any trkA-positive fibers in the dorsolateral area (Fig. 3, SI Fig. 1c), suggesting that the S100 labeling observed in this area resulted from non-crypt neurons. Within the mediodorsal area we have identified the crypt neuron target as a single glomerulus, mdg2.

Backtracing with DiI from the mdg2 glomerulus labels nearly exclusively crypt neurons. We wished to verify mdg2 as crypt neuron target glomerulus by an independent method. To this end we backtraced olfactory receptor neurons connecting to this glomerulus by localized injection of DiI, an intensely fluorescent dye, into the mdg2 glomerulus. The injection site (Fig. 4e,i) was chosen in the unlabeled olfactory bulb according to the stereotyped position of mdg2, monitored during tracing by the emergence of fluorescent axon bundles, and its coordinates were determined in the whole mount after tracing. About half of the injections ( $\mathrm{n}=$ 12) resulted in localized dye injections. We considered an injection localized, if the half-width of fluorescence intensity after tracing was $100-150 \mu \mathrm{m}$, at the lower range corresponding to about one glomerular diameter. Seven of these injections resulted in backtraced olfactory receptor neurons. Two of those injections $(0.10,0.26$ and $0.12,0.26$; anterior-posterior, medial-lateral coordinates, respectively, $c f$. SI Fig. 1f) were centered at the position of the mdg2 glomerulus $(0.07,0.27, c f$. SI Fig. 1i-j). As control we injected at a similar posterior level about one glomerular diameter further lateral $(0.25,0.52)$, a position where $\operatorname{dlg} 1$ and possibly dlg2 of the dorsolateral group are expected (SI Fig. 1i-j). The coordinates of another control injection, further anterior and very close to the midline $(0.34,0.06)$, suggest an injection into $\operatorname{mdg} 3^{17}$, the medially adjacent neighbor glomerulus of mdg2 (SI Fig. 1i-j).

After several weeks of tracing we analysed the labeled cell populations in cryostat sections of the olfactory epithelium. Between 10 and 130 cells were labeled per injection, at the upper range corresponding to a sizable fraction of neurons innervating a glomerulus ${ }^{32,33}$. Cells backtraced from mdg2 are mostly globose and exhibit an apical position within the olfactory lamellae (Fig. 4b,f,j, SI Fig. 1b,d,e,), both telltale signs of crypt neuron-like morphology ${ }^{3,6}$. In contrast, cells backtraced from the dlg1 injection site generally showed elongated shapes and more basal positions of the somata within the lamella (Fig. 4d,h,l, SI Fig. 1b,d,e). Furthermore, cells backtraced from mdg2 rarely exhibited any dendritic processes, as expected for crypt neurons, whereas cells backtraced from the more central injection site (dlg 1) mostly showed long dendritic processes, which are expected for ciliated olfactory receptor neurons (Fig. 4). Cells backtraced from 

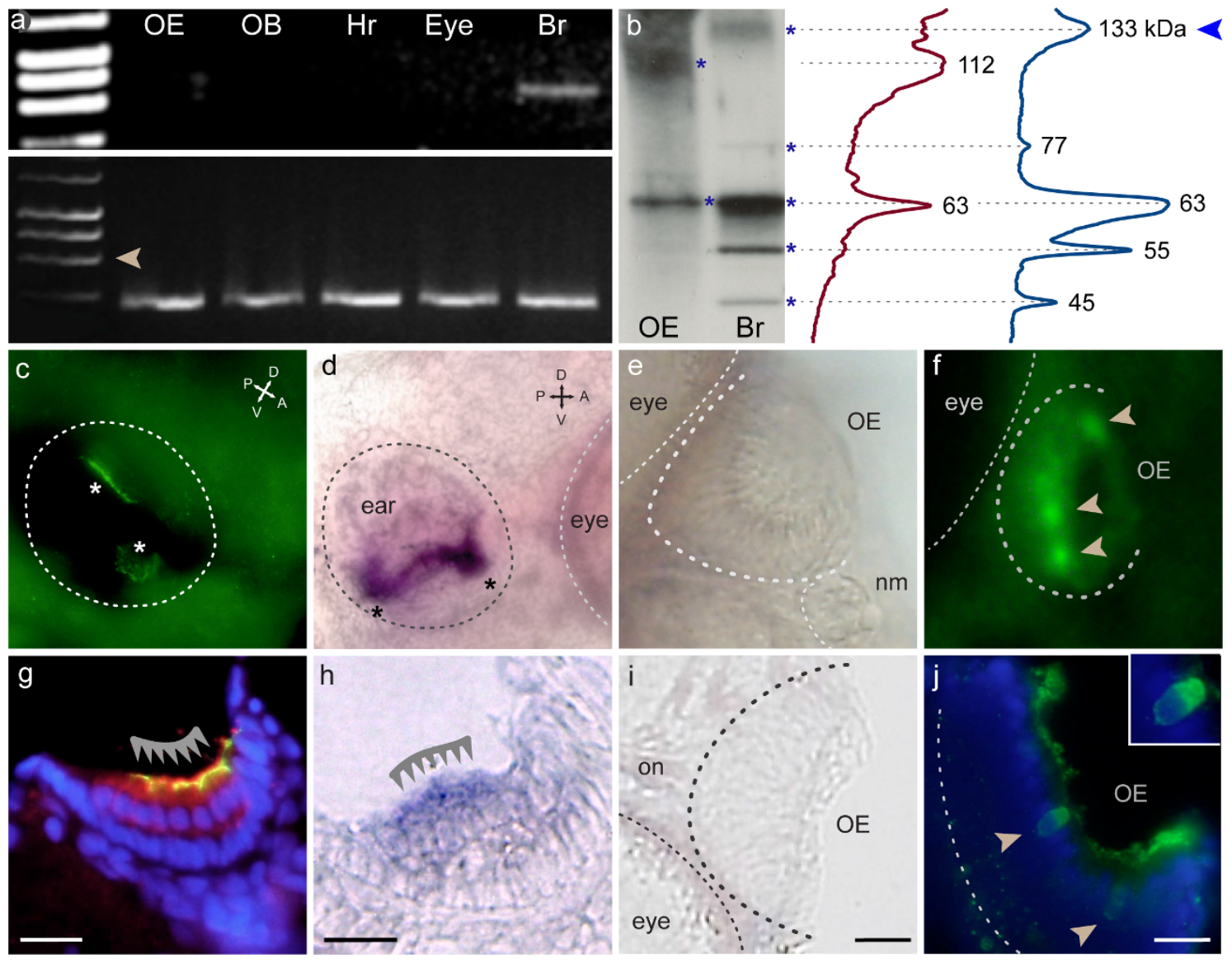

Figure $2 \mid$ TrkA-like immunoreactivity does not co-localize with TrkA expression in the olfactory epithelium. (a) RT-PCR shows TrkA expression (upper panel) in brain (Br), but not in olfactory epithelium (OE) nor olfactory bulb (OB), heart (Hr) and eye, all from adult animals. Beta actin signals (lower panel) are of similar intensity for all tissues. Arrowhead, position of amplification product from genomic DNA. (b) Left side, Western Blot with protein extracts from brain $(\mathrm{Br})$ and olfactory epthelium $(\mathrm{OE})$. Apparent molecular weight for several bands (asterisks) in brain and olfactory epithelium was determined from line scans (right side), only brain extracts show expected band (arrowhead). (c-f) Whole mounts of 5 dpf zebrafish larvae; $(g-j), 8-10 \mu \mathrm{m}$ sections from the whole mounts depicted above. Panels $(c, f, g, j)$ show TrkA antibody staining, panels (d, e, h, i) depict in situ hybridization with TrkA probe. (c) TrkA antibody staining labels several structures in the inner ear of zebrafish larvae (asterisks). (d) TrkA probe labels similar structures in the inner ear (asterisks). (e) No signal is observed with the same TrkA probe in the olfactory epithelium of the same larvae.

(f) However, TrkA antibody clearly labels several cells in the olfactory epithelium. (g) Specific labeling (barbed line) in hair cells of the macula (yellow due to complete overlap with HCS1 staining (red), a hair cell marker ${ }^{51}$. Blue, DAPI as nuclear counterstain. (h) The TrkA probe labels hair cells of the macula (barbed line). (i) No signal from TrkA probe detected in sections of the olfactory epithelium. (j) In contrast, TrkA antibody labels sparse cells in the OE with a globose morphology. Scale bars $20 \mu \mathrm{m}$ for $(\mathrm{g}-\mathrm{j})$.

the mdg3 position exhibited an intermediate morphology (SI Fig. 1e), less elongated than those from the more central injection site and less globose than crypt neurons, consistent with a microvillous phenotype.

We evaluated the significance of these findings by quantification of morphological variables for cells backtraced from mdg2 glomerulus and comparing them with the values obtained for control injections in adjacent glomeruli. The most conspicuous feature of crypt cells is their globose shape. This shape will result in smaller values for the ratio of vertical to horizontal diameter, compared to those for other types of neurons. TrkA-labeling showed 1.7 as upper limit of diameter ratios for crypt neurons (Fig. 1g) and most noncrypt cells exhibit distinctly larger values (cf. Fig. 1g, SI Fig. 1e). We therefore selected 1.5 as a conservative cutoff criterion to assign a backtraced cell to either crypt or non-crypt neuron population.

The large majority of cells backtraced from the mdg2 glomerulus form a dense cluster in a scatter plot representation of vertical and horizontal cell diameter (Fig. 5a). All cells in the cluster exhibit diameter ratios below 1.5, in other words, crypt neuron morphology. The complete distribution of diameter ratios for cells backtraced from the mdg2 glomerulus is very similar to the distribution for TrkA-labeled crypt neurons, both in the traditional histogram representation (Fig. 5b) and, even more distinctly, in the unbinned representation as empirical cumulative distribution function (Fig. 5c). The distribution of cells backtraced from mdg2 deviates from the TrkA distribution only by the presence of a small shoulder towards larger diameter ratios, presumably reflecting the presence of a small population of non-crypt neurons in the backtraced cells, cf. (Fig. 5a). Another injection at mdg2 coordinates showed very similar results, albeit with a slightly larger shoulder towards larger diameter ratios (SI Fig. 1e), presumably due to the slightly less localized nature of this injection.

In contrast, cells backtraced from control injections into dlg1 and mdg3 glomeruli show without exception diameter ratios well above 1.5 (Fig. 5a, SI Fig. 1d,e), i.e. a drastically different distribution (Fig. 5b, c, SI Fig. 1e). Pairwise comparisons of the unbinned distributions by a Kolmogorov-Smirnov test ${ }^{22}$ showed very significant differences between either control injection and TrkA-labeled crypt neurons ( $\mathrm{p}<10^{-6}$, SI Table 1 ), whereas the mdg2 injection was not significantly different (SI Table 1). Furthermore, the distribution of 

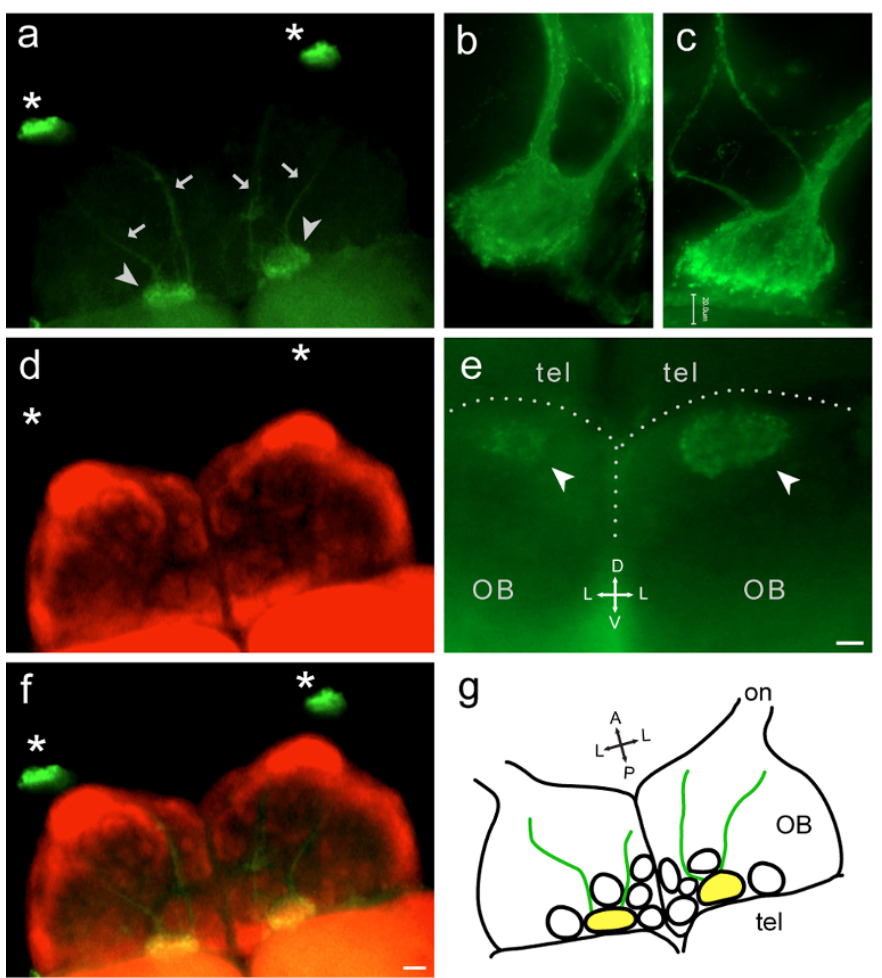

Figure 3 TrkA antibody labels a single glomerulus in the olfactory bulb. (a), (d), (f) Whole mount of adult zebrafish olfactory bulb labeled with (a) TrkA antibody (green); (d) synaptic vesicle protein 2 (SV2, red); and (f) overlay of both labels. SV2 is a general synaptic marker, which visualizes all glomeruli. The olfactory nerves were cut at the entrance to the olfactory bulb (asterisks) before staining. Dorsal view, for orientation compare the schematic drawing (g). A single labeled glomerulus (arrowhead), very far posterior and 'not-quite-medial', is seen in each olfactory bulb, entered by two nerve fascicles (arrows). Yellow color in overlay (f) confirms the TrkAlabeled structure as glomerulus. (b), (c), higher magnification of two other TrkA-labeled glomeruli, each with two incoming nerve fascicles.

(e) posterior vibratome cross section of olfactory bulbs shows the extremely dorsal position of the TrkA-labeled glomerulus. No other glomeruli are labeled by TrkA antibody in this or more anterior bulbar sections (Suppl. Fig. 1c). Scale bar $200 \mu \mathrm{m}$ for (a,d,f) and $20 \mu \mathrm{m}$ for (c,e).

cells backtraced from the mdg2 glomerulus was very significantly different from either of the two control injection sites $\left(\mathrm{p}<10^{-5}\right.$, SI Table 1).

The highly segregated distributions for mdg2 and neighboring control injections show that dye uptake has been nearly completely localized to the mdg2 glomerulus or excluded from it in the case of control injections. Any sizable spillover of dye uptake should have resulted in considerable overlap of these distributions.

As an auxillary variable we also quantified the position of the cell somata with respect to the basal-to-apical dimension. Crypt neurons exhibit a very apical location, close to the lumen, with microvillous olfactory receptor neurons situated somewhat deeper and ciliated receptor neurons more basally located, closer to the basal lamina. We find that the basal-to-apical distribution of cells backtraced from the mdg2 glomerulus is very similar to that of TrkA-labeled crypt neurons (Fig. 5d, e). A small shoulder towards more basal values (Fig. 5d,e) in the former, but not the latter presumably reflects the presence of a small population of non-crypt neurons in the backtraced cells, $c f$. (Fig. 5a). In contrast, cells backtraced from the dlg1 control injection show a drastically different basal-to-apical distribution, both with respect to peak position and steepness of the distribution (Fig. 5d,e). Kolmogorov-Smirnov test showed this difference to be highly significant $\left(\mathrm{p}<10^{-6}\right)$.
Taken together, a thorough quantitative analysis of cell morphology has shown that most cells backtraced from the mdg2 glomerulus are crypt neurons. Since we made a conservative estimate for the cutoff criterion for identification of crypt neurons, and since some diffusion of the dye outside the direct site of injection is unavoidable, resulting in a small fraction of backtraced cells that connect to adjacent glomeruli, these data support the assumption that mdg2 is innervated exclusively by crypt neurons.

\section{Discussion}

Crypt neurons have engendered considerable interest as a third type of olfactory receptor neurons with a peculiar morphology combining elements of the other two populations, ciliated and microvilllous receptor neurons ${ }^{3}$. Several attempts have been made to elucidate their neuronal circuits, beginning with studies that sought to identify their target regions in the olfactory bulb ${ }^{14-18,34}$. A cluster of mediodorsal glomeruli has been suggested as potential targets of crypt neurons, based on absence of fluorescence in some genetically labeled zebrafish lines and differential staining in others ${ }^{16}$. However, backtracing from the olfactory bulb pointed to broader target regions, as DiI injections in both dorsomedial and dorsolateral field resulted in labeled crypt neurons ${ }^{14}$. Similarly, attempts to use the crypt neuron marker S100-like immunoreactivity in the olfactory bulb clearly pointed to both dorsomedial and dorsolateral sites ${ }^{17}$, although those authors chose to focus on only one of those sites. Taken together, all these attempts to localize the target glomerulus/ glomeruli used unspecific antibodies ${ }^{14,16,17}$ or diffusible backtracing dyes $^{14,15}$, both of which might overestimate the spatial extent of the target region.

Recently we reported that a single olfactory receptor, the V1R-like ora4 gene, is expressed in all crypt neurons ${ }^{6}$ as defined by quantitative morphological assessment. This finding per se could suggest either several target glomeruli, $c f$. e.g. ${ }^{9}$ or a single target glomerulus, cf. e.g. ${ }^{8}$. A clarification of the target region critically depends on the availability of a suitable marker for crypt neurons - as shown recently ${ }^{6}$ and summarized here in the Introduction, S100-like immunoreactivity is not suitable for this purpose. Thus we set out to identify a robust and specific marker, which could be used to directly identify the crypt neuron target glomerulus/glomeruli.

We report here that a TrkA antibody previously shown to label cell somata with crypt neuron-like morphology in the olfactory epithelium $^{21}$ fulfills these criteria. We show that the TrkA antibody is fully specific under standard histological conditions that enable detection of axons and terminals, unlike the S100 antibody also suggested by the same group as crypt neuron marker ${ }^{19}$. Unfortunately, in crypt neurons the anti-TrkA antibody does not detect TrkA, but an unknown antigen, and consequently it is not straightforward to extend our results into development of a genetic marker for crypt neurons. Nevertheless we observe staining in all cellular compartments, somata, axons and axonal terminals, which allowed the unequivocal determination of the crypt cell target region.

Using this marker, we could show that crypt neurons project exclusively into a single glomerulus of the mediodorsal field, the mdg2 glomerulus. The glomerulus is named according to ${ }^{17}$ and is identical to the mdpG2 glomerulus in the original study showing stereotyped interindividually invariant glomeruli in the zebrafish olfactory bulb ${ }^{30}$. Crypt neuron termini were neither detected in the remaining glomeruli of the mediodorsal group nor in any other glomerulus. Backtracing with DiI confirmed this conclusion, since the large majority of neurons backtraced from the mdg2 glomerulus showed crypt neuron-specific morphology, whereas no such cells were labeled by backtracing from neighboring glomeruli. Our backtracing results are partially consistent with experiments by Gayoso et al, 2012 $2^{15}$, who showed labeling of crypt neurons after application of DiI to the mediodorsal glomerular field (mdg1-6), although in those studies no attempt was made to narrow down the target structure(s). 

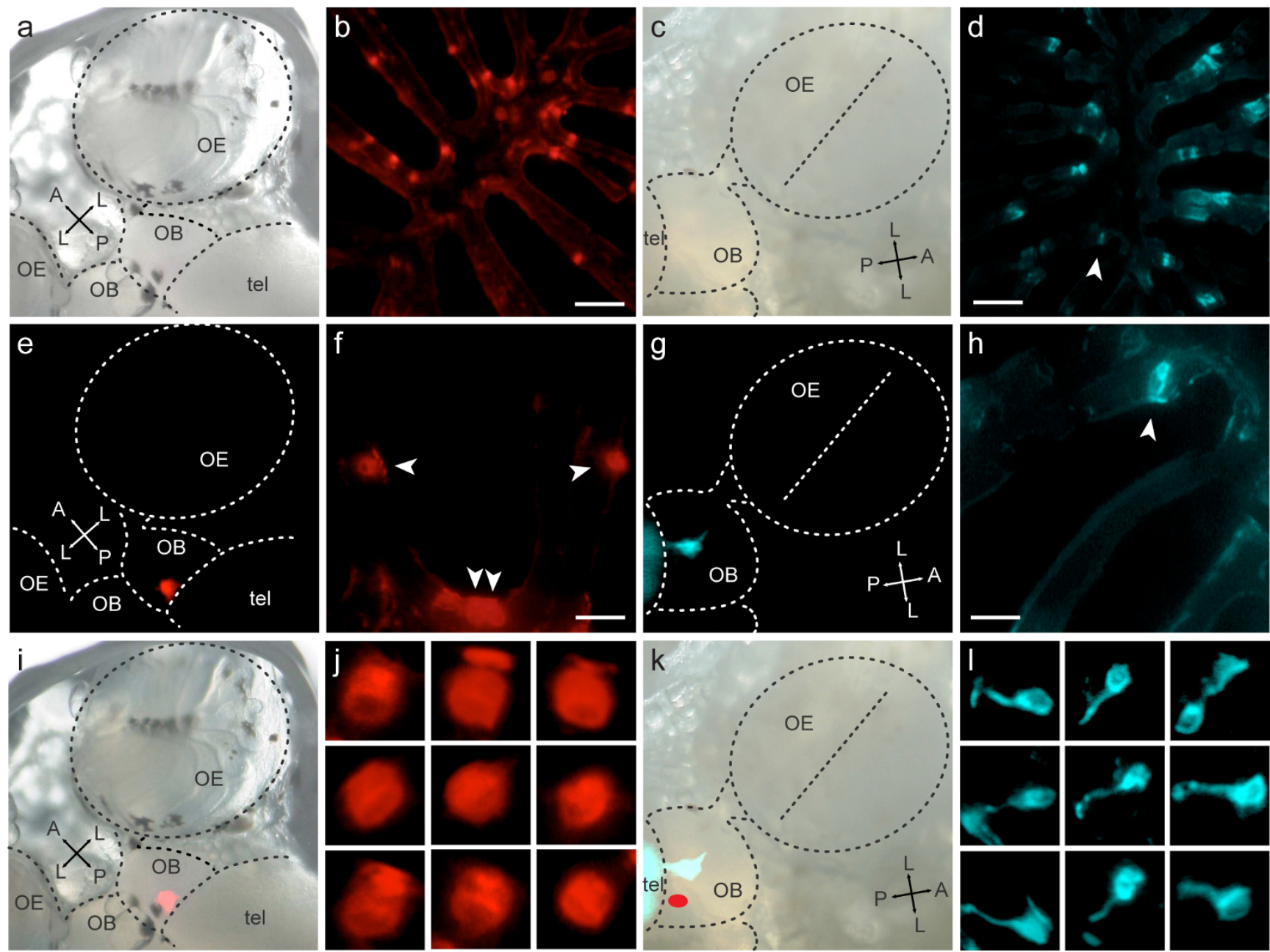

Figure $4 \mid$ DiI injection in mdg2 glomerulus labels crypt neurons, in contrast to injection in neighboring glomeruli. Localized injections of DiI were placed at the approximate position of mdg2 and dlg1 glomerulus and verified after tracing ((e), (i) and (g), (k), respectively). False color is used to distinguish mdg2 injections (red) and dlg1 injections (blue) in all panels. Results shown are from one animal each for mdg2 and dlg1 position.

(a), (c), brightfield micrographs show olfactory epithelium, olfactory bulb and anterior telencephalon. (e), (g), fluorescent images of the same area show the injection sites after the tracing period. (i), (k), overlay of brightfield and fluorescent images above shows the position of injection sites. Red oval in $\mathrm{k}$ ) represents the site of injection into mdg2. Second (mdg2 injection) and fourth (dlg1 injection) column show tracing results in cryostat sections of the olfactory epithelium. (b), (d), several cells are labeled within a section; (f), (h), enlargements from another (f) or the same section (h). (J) nine representative cells backtraced from mgd2 position from several different sections are shown at high magnification; (l). likewise for dlg1 position. Note the clear difference in morphology between cells backtraced from mdg2 and the adjacent dlg1 position. Scale bars, b,d (80 $\mu \mathrm{m})$ and (f,h) $(10 \mu \mathrm{m})$ respectively.

However, ${ }^{15}$ also reported backtracing of crypt neurons after DiI injections into the dorsolateral field (dlg glomeruli), in contrast to results from our injection into the dlg1 glomerulus. We assume that this result ${ }^{15}$ might be explainable by subtle differences in dye application leading to increased diffusion of the dye compared to our experiments. Taking into account that the more restricted pattern of backtraced cells is more likely to accurately reflect the true condition we conclude that crypt neurons in zebrafish possess a single dorsomedial target glomerulus, named mdg2 according to ${ }^{17}$.

The presence of a single target glomerulus for crypt neurons is consistent with expectations for the target size of crypt neurons, since both the sparse spatial pattern of crypt neurons ${ }^{3,6}$ and the absolute numbers of neurons labeled by either of the crypt neuron markers (TrkA, this study; ORA4, S100 in specific labeling conditions ${ }^{6}$ ) are well within the range of corresponding values for individual olfactory receptor genes ${ }^{31,33,35}$.

The very homogenous labeling of glomerular structures by the TrkA antibody would seem to argue against the small number of backtraced cells without explicit crypt neuron morphology reflecting an additional innervation to the mdg2 glomerulus. We consider it much more likely that some diffusion of DiI from the injection site into neighboring glomeruli, e.g. the very closely appositioned mdg3 glomerulus, results in labeling of some non-crypt neurons. Generally it has not been possible to restrict such dye injections to a single glomerulus ${ }^{14,34}$. Even though our injections do show glomerular resolution, a small minority of cells backtraced from adjacent glomeruli is to be expected.

Our characterisation of a second immunohistochemical marker for crypt neurons (TrkA-like immunoreactivity), independent from a previously characterized marker (S100-like immunoreactivity ${ }^{6}$ ), and nevertheless completely overlapping, strengthens the concept resulting from a preceding study ${ }^{6}$ that crypt neurons show a novel 'one cell type - one receptor' mode of expression, distinct from the 'one neuron - one receptor' mode of expression established for ciliated olfactory receptor neurons ${ }^{31,36}$. Furthermore, here we extend this observation to a 'one cell type - one receptor - one glomerulus' 

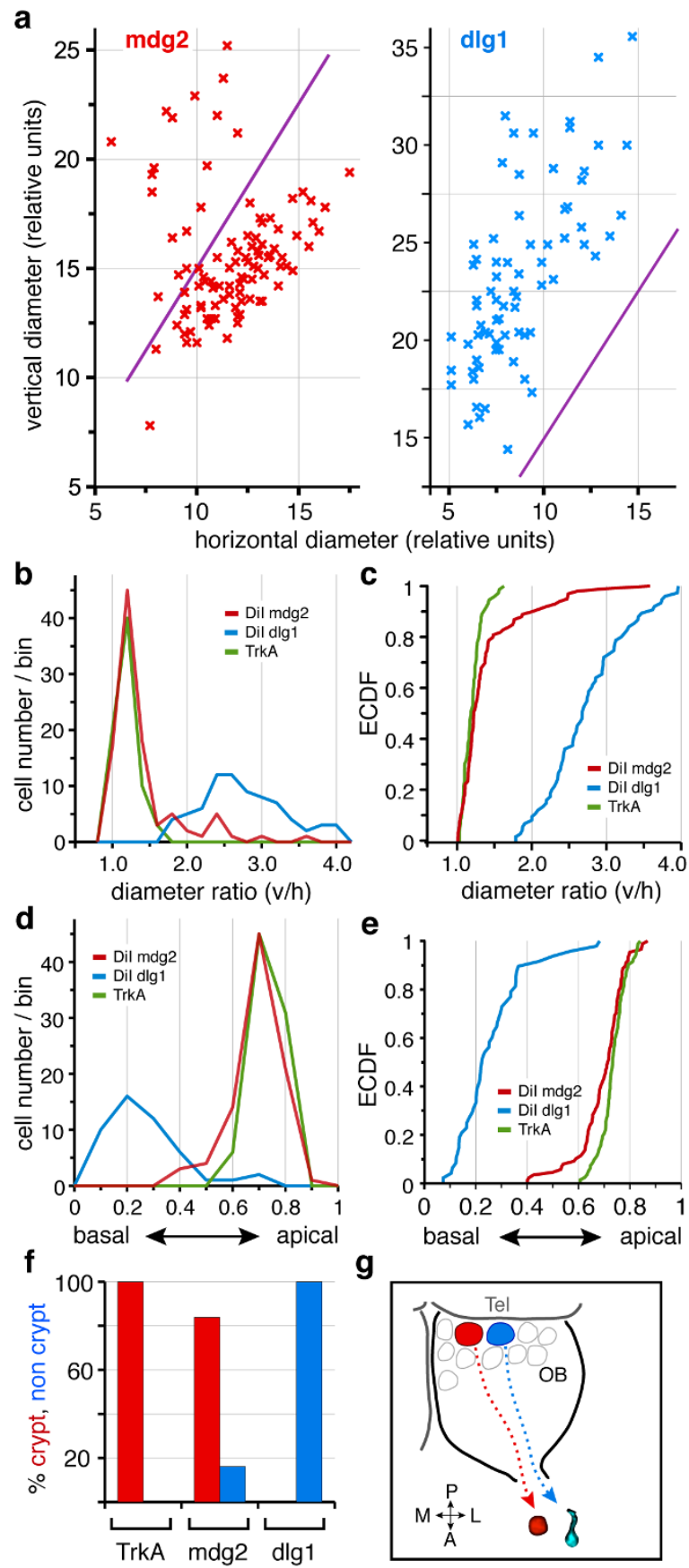

g

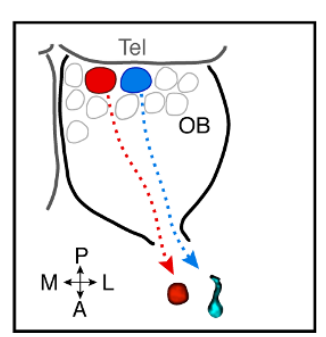

Figure 5 Quantitative analysis of tracing results shows specific crypt neuron labeling for $\mathbf{m d g} 2$ injection sites. (a) Scatter plot of vertical $v s$. horizontal diameter for injections into mdg2 (left panel) and dlg1 (right panel), each cross represents a single cell, data are from the same injections depicted in Fig. 4. Cells are considered to have crypt-like morphology, if the ratio vertical to horizontal diameter is equal or less than 1.5 (cutoff visualized by the magenta line). Note the dense cluster of crypt neurons in the mdg2 injection and the absence of crypt neurons for injections into the dlg position. (b) histogram and (c) CDF of the diameter ratios for mdg2 (red) and dlg1 (blue) injections and trkA-labeled cells (green, same values as shown in Fig. 1f). Note that distributions for mgd2 injections and TrkA, the crypt neuron marker, are indistinguishable, whereas the distribution for dlg1 sharply diverges. (d) histogram and (e) CDF of the basal-to-apical position for mdg2 (red) and dlg1 (blue) injections and trkA-labeled cells (green), same conclusion as in (b-c). (f) Percentage of cells with crypt-like diameter ratios in TrkA-labeled cells, cells backtraced from the mdg2 injection and the dlg1 injection. (g) schematic representation of the backtracing results. concept, which is reminescent of the specialized subsystems used in insect pheromone detection ${ }^{37}$ but to the best of our knowledge represents a novel coding strategy in vertebrate olfaction.

It should be noted that this interpretation rests on the assumption that the entire crypt neuron population is labeled by TrkA-like immunoreactivity. Since it is not possible to quantify crypt neuron numbers at the EM level with any degree of accuracy, $c f . .^{38}$, one could posit the existence of a 'cryptic' cell population with crypt neuron morphology, but invisible in either TrkA- or S100-antibody labeling. In particular the neighboring mediodorsal glomeruli ${ }^{17}$ could be considered candidate targets for such a 'cryptic' population, since they, like mdg2, are not labeled in zebrafish transgenic for markers of the other two known cell populations, ciliated and microvillous receptor neurons ${ }^{16}$. While we can not rule out this hypothetical possibility altogether, several observations provide contrary evidence.

Firstly, a control injection of DiI at the approximate coordinates of the mdg3 glomerulus labeled elongated cells resembling microvillous neurons, i.e. with non-crypt morphology. Secondly, $\mathrm{G}_{\mathrm{o}}$-like immunoreactivity is a specific marker for another mediodorsal glomerulus, mdg $5^{17}$, and labels cells with non-crypt morphology in the olfactory epithelium ${ }^{17}$. Thirdly, the mere existence of a small and variable proportion of non-crypt neurons in addition to the crypt neurons backtraced from injections into the mdg2 glomerulus argues against crypt neurons innervating all adjacent glomeruli, since these noncrypt (and non-ciliated) neurons presumably result from dye diffusion into the adjacent mediodorsal glomeruli.

The receptor genes expressed by neurons innervating the other five glomeruli of the mediodorsal group ${ }^{17}$ are not known. It is noteworthy that there are six genes in the V1R-like ora gene family of zebrafish $^{39}$, and six glomeruli in the mediodorsal group. Here we have paired one of these genes (ora4) with one of these glomeruli (mdg2). It remains to be seen, whether this finding might be generalizable to the entire ORA family and entire mediodorsal glomerular group.

Interestingly, for two other fish species, catfish and crucian carp, a ventral position of the crypt neuron target region has been suggested based on backtracing experiments ${ }^{34,40}$ similar to those performed here. A distinctly different position of the target glomerulus of crypt neurons could entail differences in the subsequent circuits and thus possibly differences in function of crypt neurons between species. The relatively medial position of the zebrafish crypt neuron glomerulus is consistent with the axons of downstream projection neurons joining the medial olfactory tract, $c f .{ }^{41}$ for the topology of the projection neurons. In several species the medial olfactory tract is assumed to convey pheromone detection ${ }^{42-44}$ as well as alarm response $^{11}$, and conceivably either pheromones or alarm substance might act as activators of crypt neurons. However, different ligands have been reported to activate crypt neurons ${ }^{12,13}$ and their downstream projection neurons in different species $^{34}$. So far it is not known whether this reflects a difference in receptor gene expression between species or, alternatively, species-specific alterations of the ORA4 receptor. Additionally, species differences in $G$ alpha gene expression have been reported ${ }^{6,40,45}$, which also may implicate different functions for crypt neurons of different species.

Taken together, we have identified the target region for zebrafish crypt neurons as a single glomerulus, situated dorsally and rather medially in the olfactory bulb. This extends the previous hypothesis of 'one cell type - one receptor' for crypt neurons into a 'one olfactory receptor cell type - one target glomerulus' concept, a novel coding strategy in vertebrate olfaction.

\section{Methods}

Antibodies, tissue and animal handling. Primary antibodies used were anti-S100 antibody (rabbit IgG; $1: 1000$; catalog no. Z0311, Dako), anti-TrkA (763) antibody (rabbit IgG; $1: 100 ;$ sc-118, Santa Cruz Biotechnology), anti-HCS1 monoclonal and anti-SV2 monoclonal mouse IgG1 (supernatant $1: 250$ and $1: 50$, respectively, Developmental Studies Hybridoma Bank, University of Iowa, Iowa City, IA). 
Secondary antibodies used were donkey anti-rabbit IgG conjugated to Alexa Fluor 488 (A21206, Invitrogen), goat anti-rabbit IgG conjugated to Alexa Fluor 594 (A11012, Invitrogen) and goat anti-mouse conjugated to Alexa Fluor 594 (A11005, Invitrogen).

Adult wild type zebrafish (Ab/Tü strain, 8-12 months old) were maintained at $28^{\circ} \mathrm{C}$ on $14 / 10$-hour light/dark cycle. Progeny was raised in a nursery incubator under the same conditions.

Adult fish were sacrificed by decapitation during anesthesia with MS-222 (ethyl 3aminobenzoate, Sigma). Animal handling was covered by Animal Use Record 20.10.217 (issued by the State Agency for Nature, Environment and Consumer Protection NRW, LANUV).

Tissues were embedded in 5\% low melting agarose and sectioned by vibratome (Pelco 101) or embedded in TissueTek O.C.T. compound (Sakura), and cut by cryostat (Leica CM1900) at $-20^{\circ} \mathrm{C}$. Fluorescence was analysed using wide field fluorescence microscopes (Keyence BZ-8100E and BZ-9000) for sections and a Nikon CoolPix 950 digital camera attached to a Nikon SMZ-U binocular for whole mounts.

Whole mount olfactory bulb immunohistochemistry. The dorsal cranium was removed, exposed brains were fixed by immersion in $4 \%$ paraformaldehyde (PFA, $\mathrm{pH}$ 7.4) in phosphate-buffered saline (PBS, pH 7.5) overnight at $4^{\circ} \mathrm{C}$ and olfactory bulbs still connected to telencephalon were dissected out. Staining was performed according to ${ }^{17}$, with minor modifications. After blocking, samples were incubated with primary antibodies anti-TrkA and anti-synaptic vesicle protein 2 (SV2) at $4{ }^{\circ} \mathrm{C}$ for 20 to 25 days on a vertical rotator ( $5 \mathrm{sec} /$ round), followed by several washes over a period of $3 \mathrm{hrs}$ at room temperature. Subsequently, the olfactory bulbs were incubated with secondary antibodies for 7 days at $4{ }^{\circ} \mathrm{C}$, followed by several washes at room temperature. Tissue was cleared as described ${ }^{17}$. Both primary and secondary antibodies were used at a final dilution of $1: 100$ in blocking reagent. For detailed examination $100 \mu \mathrm{m}$ vibratome sections were analysed.

Whole mount larvae immunohistochemistry. 10-20 5dpf embryos were fixed overnight in 4\% PFA in PBS, washed in PBS and permeabilized overnight with $1.5 \%$ Triton-X 100 in $1 \times$ PBS at $4{ }^{\circ} \mathrm{C}$, followed by 2 hrs blocking at room temperature in $\mathrm{BDP}(0.1 \%$ DMSO, $1 \% \mathrm{BSA}$ in $1 \times$ PBS) containing $5 \%$ NGS (Normal goat serum). The larvae were incubated with a cocktail of primary antibodies in BDP overnight at $4^{\circ} \mathrm{C}$, followed by multiple washes in $\mathrm{BDP}$ at room temperature. Larvae were incubated with secondary antibodies $\left(1: 250\right.$ dilution) in BDP overnight at $4{ }^{\circ} \mathrm{C}$, followed by extensive washes with BDP over a period of several hours with multiple changes; subsequently the BDP was exchanged with $1 \times$ PBS. The success of the staining was confirmed using a fluorescent microscope, subsequently cryosections of $8 \mu \mathrm{M}$ thickness were prepared. Slides were mounted with VectaShield containing DAPI (Vector)

Immunohistochemistry on cryosections. Heads were either pre-incubated before dissection overnight at $4{ }^{\circ} \mathrm{C}$ in freshly prepared $4 \%$ PFA in $1 \times \mathrm{PBS}$ (pre-fixed tissue) or dissected directly (fresh-frozen tissue). Horizontal cryosections $(8 \mu \mathrm{m})$ of the olfactory epithelia were thaw-mounted onto Superfrost Plus slide glasses (Thermo), incubated in acetone at $-20^{\circ} \mathrm{C}$ for 15 mins, washed several times in PBST, and blocked in 5\% normal goat serum in PBST (blocking solution) for $1 \mathrm{hr}$ at room temperature. In order to overcome the limitations arising from same species antibodies in double labeling, the $\mathrm{Fc}_{\mathrm{c}}$ portion of the anti-S100 antibody was covalently conjugated with fluorescein (Thermo Scientific, 53029) as described ${ }^{46}$. For double labeling, the slides were overnight incubated at $4{ }^{\circ} \mathrm{C}$ with anti-TrkA antibody $(1: 200$ dilution in blocking solution), washed 3 times in PBST to remove unbound antiTrKA and incubated for $2 \mathrm{hrs}$ at room temperature with the first of the two secondary antibodies (anti-rabbit alexa fluor 488). Slides were further washed 3 times in PBST, incubated for $1 \mathrm{hr}$ in blocking solution, followed by overnight incubation at $4{ }^{\circ} \mathrm{C}$ with flu-labeled anti-S100 (second primary antibody), washed 3 times for $10 \mathrm{~min}$ each and incubated for $2 \mathrm{hrs}$ at room temperature with alkaline phosphatase (AP) conjugated anti-fluorescein (the second of the two secondary antibodies). S100-labeled cells were visualized by enzymatic reaction of AP with HNPP Fluorescent Detection Set (Roche). The slides were washed in PBS and mounted with VectaShield containing DAPI (Vector).

Western blot analysis. Olfactory epithelium and telencephalon from six fishes were dissected and immediately transferred to RIPA lysis buffer (Sigma, R0278), followed by mechanical homogenization and $10 \mathrm{~min}$ sonication. The samples were centrifuged for 10 mins at $4^{\circ} \mathrm{C}$ and the protein concentration in the supernatant was determined by Bradford. Protein samples were separated by SDS-PAGE and transferred onto PVDF membrane by electrophoretic transfer (100 V, $90 \mathrm{~min})$. Afterwards, the membrane was washed 3 times with PBS containing $0.1 \%$ Tween 20 for 5 minutes each and blocked using 5\% milk powder (BIO-RAD, 170-6404) dissolved in PBST for 1 hour at room temperature. Primary antibody was prepared in 5\% skim milk (DNA grade, BioRad) in PBST and added to the membrane followed by incubation at $4{ }^{\circ} \mathrm{C}$ overnight. After 3 washes for 5 minutes each in PBST, the membrane was incubated in secondary antibody, prepared in 5\% skim milk in PBST for 1 hour at room temperature. After three rinses in PBST, ECL reagent plus Western Blotting Detection Reagents (RPN2132) was used for developing. Western blots were analysed using ImageJ (http://rsbweb.nih.gov/ij/).

RT-PCR. Total RNA samples were prepared from adult zebrafish tissues of the wildtype Ab/Tübingen strain with the RNeasy kit (QIAGEN). After digestion with DNase
I, 100 ng RNA for each tissue were subjected to the first-strand cDNA synthesis with RevertAid MmLV reverse transcriptase (Fermentas), using oligo(dT) ${ }_{15}$ primer. Subsequent PCR was performed using Red Taq mix (Bioline) with gene-specific primers for Dr_actin and Dr_TrkA (forward: CCCCATTGAGCACGGTATT, reverse: TCATGGAAGTCCACATGGCAGAAG, and forward: ACTTTGAAAATAGCCAATGAGTCC, reverse: TGATGACCAACCTTTGCTGT, respectively).

The following conditions were used: $10 \mathrm{~min}$ at $95^{\circ} \mathrm{C}$, followed by 35 cycles of $45 \mathrm{sec}$ at $95^{\circ} \mathrm{C}, 45 \mathrm{sec}$ at $55^{\circ} \mathrm{C}$, and $60 \mathrm{sec}$ at $72^{\circ} \mathrm{C}$, and a final extension of $10 \mathrm{~min}$ at $72^{\circ} \mathrm{C}$

Whole mount in situ hybridization. Digoxigenin (DIG) RNA probes were synthesized according to the DIG RNA labeling kit supplier protocol (Roche Molecular Biochemicals) using the Dr_TrkA primers.

FW $5^{\prime}$ - AAGGTACC GGCTGAATGTGCCAATCTCT $-3^{\prime}$ and RV 5' AAGAGCTCTCCCCGATCTTCACTACCAG - $3^{\prime}$. In situ hybridization was carried out according to ${ }^{47}$. Hybridizations were performed on $5 \mathrm{dpf}$ old larvae overnight at $65^{\circ} \mathrm{C}$ and stringent washes were done in $0.2 \times \mathrm{SSC}$ at $65^{\circ} \mathrm{C}$. Anti-DIG primary antibody coupled to alkaline phosphatase (Roche Molecular Biochemicals) and NBTBCIP (Roche Molecular Biochemicals) were used for signal detection.

DiI tracing. The fluorescent carbocyanine dye 1,1'-dioctadecyl 3,3,3',3'tetramethylindocarbocyanine perchlorate (DiI; Molecular Probes) was used for retrograde tracing of olfactory receptor neurons as described by ${ }^{32}$. Briefly, zebrafish heads were pre-fixed in freshly prepared 4\% PFA overnight. Afterwards, the dorsal side of the olfactory bulbs was exposed and a small DiI crystal was placed with the help of a glass micro-needle for 5-10 seconds. Tracing was allowed to proceed in 4\% PFA in PBS at $37^{\circ} \mathrm{C}$ for $2-3$ weeks. The fixative was changed every $2-3$ days. After tracing, olfactory epithelia, and olfactory bulbs plus telencephalon were dissected. Cryosections of olfactory epithelia were immediately analyzed. The olfactory bulb was documented as whole mount, and afterwards sectioned by vibratome (Pelco 101; $100 \mu \mathrm{m}$ transverse sections). Sections were mounted on Superfrost Plus slide glasses.

Measurement and analysis of spatial coordinates. Spatial coordinates were measured in arbitrary units and normalized. For olfactory bulb coordinates whole mounts of olfactory bulb, with telencephalon attached, were viewed from dorsal. An axis cross was put at the center of the glomerulus or injection site and maximal values were determined for medial-to-lateral and anterior-to-posterior direction as the corresponding line segments (see SI Fig. 1f). Thus possible values range from $0 / 0$ (medial most/posterior-most) to 1/1 (lateral-most/anterior-most). Vertical cell diameter was determined as maximal cell length perpendicular to the basal lamina (soma and dendrite, if any) and horizontal diameter as maximal cell width, i.e. parallel to the basal lamina (see SI Fig. 1a). For apical-to-basal position in the olfactory epithelium the shortest distance between center of the cell soma and basal border of the epithelial layer was normalized to the shortest distance between basal and apical border of the epithelial layer at the position of the cell to be measured (see SI Fig. 1b). Thus the range of values is between 0 (most basal) and 1 (most apical).

Unbinned distributions were represented as the corresponding empirical cumulative distribution function $(\mathrm{ECDF})^{48,49}$. To estimate, whether two spatial distributions were significantly different, we performed Kolmogorov-Smirnov tests on the unbinned distributions as described in ${ }^{22}$. This test is particularly suitable for continuous distributions and makes no assumptions about the nature of the distributions investigated. This is essential since the skewness of some observed distributions showed that these are not Gaussian. Due to the sensitive nature of the test on large distributions $(\mathrm{n}>100)$ we selected $\mathrm{p}<0.01$ as cutoff criterion for significant difference. Results of the Kolmogorov-Smirnov test were confirmed by permutation analysis ${ }^{50}$ without exception.

1. Mori, K. \& Sakano, H. How is the olfactory map formed and interpreted in the mammalian brain? Annual review of neuroscience 34, 467-499 (2011).

2. Mombaerts, P. Axonal wiring in the mouse olfactory system. Annual review of cell and developmental biology 22, 713-737 (2006).

3. Hansen, A. \& Zeiske, E. The peripheral olfactory organ of the zebrafish, Danio rerio: an ultrastructural study. Chemical senses 23, 39-48 (1998).

4. Ferrando, S. et al. Observations of crypt neuron-like cells in the olfactory epithelium of a cartilaginous fish. Neuroscience letters 403, 280-282 (2006).

5. Hansen, A. \& Finger, T. E. Phyletic distribution of crypt-type olfactory receptor neurons in fishes. Brain, behavior and evolution 55, 100-110 (2000).

6. Oka, Y., Saraiva, L. R. \& Korsching, S. I. Crypt neurons express a single V1Rrelated ora gene. Chemical senses 37, 219-227 (2012).

7. Mombaerts, P. et al. Visualizing an olfactory sensory map. Cell 87, 675-686 (1996).

8. Johnson, M. A. et al. Neurons expressing trace amine-associated receptors project to discrete glomeruli and constitute an olfactory subsystem. Proceedings of the National Academy of Sciences of the United States of America 109, 13410-13415 (2012).

9. Wagner, S., Gresser, A. L., Torello, A. T. \& Dulac, C. A multireceptor genetic approach uncovers an ordered integration of VNO sensory inputs in the accessory olfactory bulb. Neuron 50, 697-709 (2006).

10. Hamdani el, H., Lastein, S., Gregersen, F. \& Doving, K. B. Seasonal variations in olfactory sensory neurons--fish sensitivity to sex pheromones explained? Chemical senses 33, 119-123 (2008). 
11. Mathuru, A. S. et al. Chondroitin fragments are odorants that trigger fear behavior in fish. Current biology: CB 22, 538-544 (2012).

12. Vielma, A., Ardiles, A., Delgado, L. \& Schmachtenberg, O. The elusive crypt olfactory receptor neuron: evidence for its stimulation by amino acids and cAMP pathway agonists. The Journal of experimental biology 211, 2417-2422 (2008).

13. Bazaes, A. \& Schmachtenberg, O. Odorant tuning of olfactory crypt cells from juvenile and adult rainbow trout. The Journal of experimental biology 215, 17401748 (2012).

14. Gayoso, J. A., Castro, A., Anadon, R. \& Manso, M. J. Differential bulbar and extrabulbar projections of diverse olfactory receptor neuron populations in the adult zebrafish (Danio rerio). The Journal of comparative neurology 519, 247-276 (2011).

15. Gayoso, J., Castro, A., Anadon, R. \& Manso, M. J. Crypt cells of the zebrafish Danio rerio mainly project to the dorsomedial glomerular field of the olfactory bulb. Chemical senses 37, 357-369 (2012).

16. Sato, Y., Miyasaka, N. \& Yoshihara, Y. Mutually exclusive glomerular innervation by two distinct types of olfactory sensory neurons revealed in transgenic zebrafish. The Journal of neuroscience: the official journal of the Society for Neuroscience 25, 4889-4897 (2005).

17. Braubach, O. R., Fine, A. \& Croll, R. P. Distribution and functional organization of glomeruli in the olfactory bulbs of zebrafish (Danio rerio). The Journal of comparative neurology 520,2317-2339, Spc2311 (2012).

18. Koide, T. et al. Olfactory neural circuitry for attraction to amino acids revealed by transposon-mediated gene trap approach in zebrafish. Proceedings of the National Academy of Sciences of the United States of America 106, 9884-9889 (2009).

19. Germana, A. et al. S100 protein-like immunoreactivity in the crypt olfactory neurons of the adult zebrafish. Neuroscience letters 371, 196-198 (2004).

20. Kraemer, A. M., Saraiva, L. R. \& Korsching, S. I. Structural and functional diversification in the teleost S100 family of calcium-binding proteins. $B M C$ evolutionary biology 8, 48 (2008).

21. Catania, S. et al. The crypt neurons in the olfactory epithelium of the adult zebrafish express TrkA-like immunoreactivity. Neuroscience letters 350, 5-8 (2003).

22. Press, W. H. T. S., Vetterling, W. T. \& Flannery, B. P. Numerical recipes in C: The art of scientific computing. Vol. second (1992).

23. Martin, S. C., Marazzi, G., Sandell, J. H. \& Heinrich, G. Five Trk receptors in the zebrafish. Developmental biology 169, 745-758 (1995)

24. Korsching, S., Auburger, G., Heumann, R., Scott, J. \& Thoenen, H. Levels of nerve growth factor and its mRNA in the central nervous system of the rat correlate with cholinergic innervation. The EMBO journal 4, 1389-1393 (1985).

25. Schimmang, T. et al. Survival of inner ear sensory neurons in trk mutant mice. Mechanisms of development 64, 77-85 (1997).

26. Catania, S. et al. Neurotrophin and Trk neurotrophin receptors in the inner ear of Salmo salar and Salmo trutta. Journal of anatomy 210, 78-88 (2007).

27. Sandulescu, C. M., Teow, R. Y., Hale, M. E. \& Zhang, C. Onset and dynamic expression of $\mathrm{S} 100$ proteins in the olfactory organ and the lateral line system in zebrafish development. Brain research 1383, 120-127 (2011).

28. Germana, A. et al. Neurotrophin receptors in taste buds of adult zebrafish (Danio rerio). Neuroscience letters 354, 189-192 (2004)

29. Nagao, H., Yoshihara, Y., Mitsui, S., Fujisawa, H. \& Mori, K. Two mirror-image sensory maps with domain organization in the mouse main olfactory bulb. Neuroreport 11, 3023-3027 (2000).

30. Baier, H. \& Korsching, S. Olfactory glomeruli in the zebrafish form an invariant pattern and are identifiable across animals. The Journal of neuroscience: the official journal of the Society for Neuroscience 14, 219-230 (1994).

31. Sato, Y., Miyasaka, N. \& Yoshihara, Y. Hierarchical regulation of odorant receptor gene choice and subsequent axonal projection of olfactory sensory neurons in zebrafish. The Journal of neuroscience: the official journal of the Society for Neuroscience 27, 1606-1615 (2007)

32. Baier, H., Rotter, S. \& Korsching, S. Connectional topography in the zebrafish olfactory system: random positions but regular spacing of sensory neurons projecting to an individual glomerulus. Proceedings of the National Academy of Sciences of the United States of America 91, 11646-11650 (1994)

33. Weth, F., Nadler, W. \& Korsching, S. Nested expression domains for odorant receptors in zebrafish olfactory epithelium. Proceedings of the National Academy of Sciences of the United States of America 93, 13321-13326 (1996).

34. Hamdani el, H. \& Doving, K. B. Specific projection of the sensory crypt cells in the olfactory system in crucian carp, Carassius carassius. Chemical senses 31, 63-67 (2006).

35. Hussain, A., Saraiva, L. R. \& Korsching, S. I. Positive Darwinian selection and the birth of an olfactory receptor clade in teleosts. Proceedings of the National Academy of Sciences of the United States of America 106, 4313-4318 (2009).
36. Barth, A. L., Dugas, J. C. \& Ngai, J. Noncoordinate expression of odorant receptor genes tightly linked in the zebrafish genome. Neuron 19, 359-369 (1997).

37. Hildebrand, J. G. Analysis of chemical signals by nervous systems. Proceedings of the National Academy of Sciences of the United States of America 92, 67-74 (1995).

38. Hansen, A. \& Zielinski, B. S. Diversity in the olfactory epithelium of bony fishes: development, lamellar arrangement, sensory neuron cell types and transduction components. Journal of neurocytology 34, 183-208 (2005).

39. Saraiva, L. R. \& Korsching, S. I. A novel olfactory receptor gene family in teleost fish. Genome research 17, 1448-1457 (2007).

40. Hansen, A. et al. Correlation between olfactory receptor cell type and function in the channel catfish. The Journal of neuroscience: the official journal of the Society for Neuroscience 23, 9328-9339 (2003).

41. Miyasaka, N. et al. From the olfactory bulb to higher brain centers: genetic visualization of secondary olfactory pathways in zebrafish. The Journal of neuroscience: the official journal of the Society for Neuroscience 29, 4756-4767 (2009).

42. Doving, K. B. \& Selset, R. Behavior patterns in cod released by electrical stimulation of olfactory tract bundlets. Science (New York, N.Y.) 207, 559-560 (1980).

43. Sorensen, P. W., Hara, T. J. \& Stacey, N. E. Sex pheromones selectively stimulate the medial olfactory tracts of male goldfish. Brain research 558, 343-347 (1991).

44. Friedrich, R. W. \& Korsching, S. I. Chemotopic, combinatorial, and noncombinatorial odorant representations in the olfactory bulb revealed using a voltage-sensitive axon tracer. The Journal of neuroscience: the official journal of the Society for Neuroscience 18, 9977-9988 (1998).

45. Hansen, A., Anderson, K. T. \& Finger, T. E. Differential distribution of olfactory receptor neurons in goldfish: structural and molecular correlates. The Journal of comparative neurology 477, 347-359 (2004).

46. Korsching, S. \& Thoenen, H. Two-site enzyme immunoassay for nerve growth factor. Methods in enzymology 147, 167-185 (1987)

47. Oka, Y., Saraiva, L. R., Kwan, Y. Y. \& Korsching, S. I. The fifth class of Galpha proteins. Proceedings of the National Academy of Sciences of the United States of America 106, 1484-1489 (2009).

48. Wilk, M. B. \& Gnanadesikan, R. Probability plotting methods for the analysis of data. Biometrika 55, 1-17 (1968).

49. Feller, W. An introduction to Probability Theory and its Applications. Vol. II chap I.12 (Wiley, 1966).

50. Manly, B. Randomization, bootstrap and Monte Carlo methods in biology (Chapman and Hall/CRC, London, 1997).

51. Goodyear, R. J. et al. Identification of the hair cell soma-1 antigen, HCS-1, as otoferlin. Journal of the Association for Research in Otolaryngology: JARO 11, 573-586 (2010)

\section{Acknowledgements}

We thank Vladimir Shiriagin for providing initial larval TrkA stainings. We gratefully acknowledge financial support from the German Science foundation (grant KO 1046/7-1 to S.I.K.) and the International Graduate School IGSDHD (G.A.).

\section{Author contributions}

The experiments were designed by S.I.K., G.A. and Y.O. and performed by G.A., I.I., M.S., Y.O. and S.I.K., S.I.K., I.I. and G.A. drafted the illustrations. Data analysis was done by S.I.K., W.N. and G.A., S.I.K. wrote the paper.

\section{Additional information}

Supplementary information accompanies this paper at http://www.nature.com/ scientificreports

Competing financial interests: The authors declare no competing financial interests.

How to cite this article: Ahuja, G. et al. Zebrafish crypt neurons project to a single, identified mediodorsal glomerulus. Sci. Rep. 3, 2063; DOI:10.1038/srep02063 (2013).

(c) (i) $\Theta$ This work is licensed under a Creative Commons Attribution-

visit http://creativecommons.org/licenses/by-nc-nd/3.0 